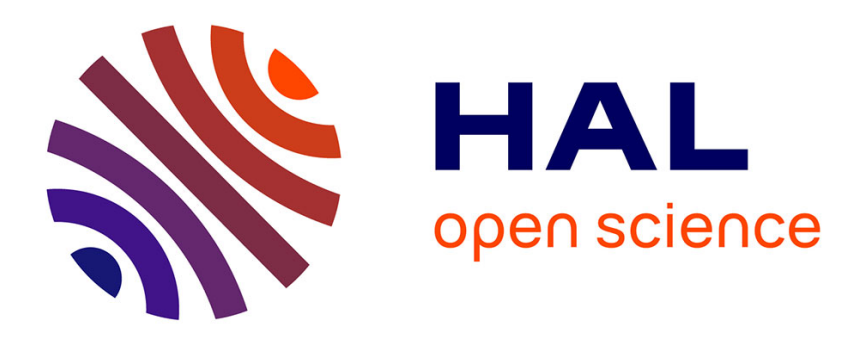

\title{
Mechanical stress relaxation in molecular self-assembly
}

Lucas Menou, Martin Castelnovo

\section{To cite this version:}

Lucas Menou, Martin Castelnovo. Mechanical stress relaxation in molecular self-assembly. Soft Matter, 2019, 10.1039/C9SM00761J . hal-02348868

\section{HAL Id: hal-02348868 \\ https://hal.science/hal-02348868}

Submitted on 5 Nov 2019

HAL is a multi-disciplinary open access archive for the deposit and dissemination of scientific research documents, whether they are published or not. The documents may come from teaching and research institutions in France or abroad, or from public or private research centers.
L'archive ouverte pluridisciplinaire HAL, est destinée au dépôt et à la diffusion de documents scientifiques de niveau recherche, publiés ou non, émanant des établissements d'enseignement et de recherche français ou étrangers, des laboratoires publics ou privés. 


\title{
Mechanical stress relaxation in molecular self-assembly
}

\author{
Lucas Menou and Martin Castelnovo \\ Univ Lyon, Ens de Lyon, Univ Claude Bernard, \\ CNRS, Laboratoire de Physique, F-69342 Lyon, France
}

(Dated: June 27, 2019)

\begin{abstract}
Molecular self-assembly on a curved substrate leads to the spontaneous inclusion of topological defects in the growing bidimensional crystal, unlike assembly on a flat substrate. We propose in this work a quantitative mechanism for this phenomenon by using standard thin shell elasticity. The Gaussian curvature of the substrate induces large in-plane compressive stress as the surface grows, in particular at the rim of the assembly, and the addition of a single defect relaxes this mechanical stress. We found out that the value of azimutal stress at the rim of the assembly determines the prefered directions for defect nucleation. These results are also discussed as function of different defect combinations, like dislocations and grain boundaries or scars. In particular, the elastic model permits to compare quantitatively the ability of various defects to relax mechanical stress. Moreover, these findings allows to understand the progressive buidling-up of the typical disclinations and grain boundaries pattern observed for ground states of large 2D spherical crystals.
\end{abstract}

Individual molecules are able to acquire collective behaviors by self-assembling, therebye reducing significantly their free energy. This behavior is widely observed across biological systems, forming structures of different spatial dimensionality (from 1D to 3D) and different shapes[1, 2]. In two dimensions, such structures are for example fluid bilayer membrane made of phospholipids, or viral capsids which are closed shells made of proteins. In this last example, icosahedral symmetry is mostly observed, especially for small viruses [3, 4]. From a local point of view, proteins are packed hexagonally and form "hexamers", almost anywhere on the surface of the virus, except at twelve locations where they form pentamers. Within the context of topology, these pentamers are considered as particular topological defects, known as disclinations. Their topological charge is positive, and negative charges correspond to heptamers with seven subunits being packed locally [5]. The presence and the net topological charge of these defects in a closed shell is mandatory $(+12)$ from a global point of view, as it is shown by Euler relation in order to satisfy the correct balance in the number of vertices, edges and faces of the associated triangular network [6-8]. However the physical mechanism associated to the progressive arising of these defects for the growth on a curved substrate is still far from clear.

From a purely local geometric point of view, using equilateral triangles to build a regular hexagon results in a flat surface, while using the same triangles to build a regular pentagon leads to a buckled structure. This shows how incorporation of pentamers in a growing surface made of hexamers amounts to produce localized net curvature for the surface, and therefore it definitely helps to curve and to close it, but it does not predict the precise location of the defects. The answer to this question is to be found by considering the elastic properties of the growing surface. Indeed, it has been observed that curved molecular surfaces are mainly produced either by using spontaneous curvature for the building blocks [9-14], or by using an additional scaffold on which the growing sur- face is adsorbed $[7,8,15-17]$. In a recent theoretical work, Li et al. have studied both analytically and numerically the energetics of fivefold defects for a surface growing onto a spherical scaffold [18]. They showed that, upon assuming irreversibility for the assembly, these defects arise as local minima of total elastic energy, and that these minima are localized at the vertices of an icosahedron, in perfect agreement with self-assembly simulations. Yet, this result is obtained from a global point of view, as all points of the surface contribute to the total energy. It is not clear from the single building block point of view what determines the favorable localization of the defects: at this length scale, the building blocks are sensible to local interactions or forces, rather than the total energy.

On the other hand, for large triangulated structures that are closed, it has been shown that the ground state configuration involves not only twelve isolated disclinations, but also a combination of fivefold and sevenfold defects, the net topological charge being conserved $(+12)$ [6]. More precisely, a single pair of fivefold-sevenfold defects forms a dipole-like type of topological defect called a dislocation, and alignments of several of these defects form grain boundaries or scars [16]. Ground state configuration has been shown to consist in twelve fivefold defects located on the vertices of an icosahedron, together with a large number of scars. In this case, it is not clear whether these configurations can be obtained through self-assembly.

We propose in this work a mechanism for defect nucleation based on the analysis of mechanical stress at the rim of the growing surface. We demonstrate in particular that the fivefold defects arise in regions where the azimuthal stress (or hoop stress) $\sigma_{\theta \theta}$ becomes negative during self-assembly, forcing therefore the local closure of pentagons instead of hexagons. The presence of compressive hoop stress has already been used in the literature to explain the wrinkles observed in thin sheets forced to adhere on spheres [19-21]. In these works, a compressionfree model was proposed, in which the hoop stress van- 
ishes in the outer regions of the surface thanks to some defect distributions. Notice that both compression free relaxing axisymmetry and numerical model without axisymmetry assumption have been used in order to reach conclusions about the optimal bulk defect distribution relaxing mechanical stress. In our work, the physics of defect nucleation through stress relaxation is similar. In particular, we do not assume any axisymmetry, allowing therefore to have a local comparison of stress relaxation patterns. In the next section, the elastic model is presented, and it is used to compute pattern of stress relaxation for different configurations. The comparison of these patterns is discussed in the last part of the article. Moreover, we provided additional datas in a supplemental material file.

\section{ELASTIC MODEL FOR GROWTH ON A CURVED SUBSTRATE}

In order to describe the elastic properties of a molecular surface growing on a curved scaffold, we have to estimate the elastic strain and stress within this surface as compared to equivalent flat geometry. We consider therefore as a reference state a flat disc of radius $R$ and thickness $h<<R$. We then force this disc to cover part of a sphere of radius $R_{0}$. Following the classical framework of plate elasticity, which we briefly summarize below, such a deformation can be reached by the use of external loads $[22,23]$. Using polar coordinates $\{r, \theta, z\}$, the deformation is first characterized by the displacement of each point from the reference state $\left\{u_{r}, u_{\theta}, w\right\}$, which is also associated with elastic strains $u_{i j}$ and stresses $\sigma_{i j}$. For large deflection of the disc, we assume the following non linear strain-displacement relationships (vonKarman strain)

$$
u_{i j}=\frac{1}{2}\left(\frac{\partial u_{i}}{\partial x_{j}}+\frac{\partial u_{j}}{\partial x_{i}}\right)+\frac{1}{2} \frac{\partial w}{\partial x_{i}} \frac{\partial w}{\partial x_{j}}
$$

which allows coupling of in-plane and out-of-plane deformations. For an isotropic material, the relation between strain and stress tensors is given by Hooke's law. Next, the state of mechanical equilibrium of the system is described using local force balance equations involving the stress tensor $\partial_{j} \sigma_{i j}=0$. Finally, a compatibility condition between the strain tensor elements has to be fulfilled in order to guarantee a unique relation between the displacements and the strain components. Introducing Airy stress function $\chi(r, \theta)$ defined by $\sigma_{i j}=\epsilon_{i k} \epsilon_{j l} \partial_{k} \partial_{l} \chi$ with $\epsilon_{i j}$ is the antisymetric unit tensor, and focusing on vertical external loads only $p(r, \theta)$, all these equations are rewritten in the form of the two famous non-linear Föpplvon Karman equations (FvK) for the Airy stress function $\chi(r, \theta)$ and the vertical deformation $w(r, \theta)[23]$ :

$$
\begin{aligned}
\frac{\nabla^{4} \chi}{Y} & =-\frac{1}{2} L(w, w) \\
D \nabla^{4} w & =L(w, \chi)+p(r, \theta)
\end{aligned}
$$

where $Y$ is the $2 \mathrm{D}$ young modulus, $D$ is bending rigidity, and $\nu$ is the Poisson's ratio. In these equations, the operator $L$ is defined as

$$
\begin{aligned}
L(w, \chi)= & \frac{\partial^{2} w}{\partial r^{2}}\left(\frac{1}{r} \frac{\partial \chi}{\partial r}+\frac{1}{r^{2}} \frac{\partial^{2} \chi}{\partial \theta^{2}}\right) \\
& +\frac{\partial^{2} \chi}{\partial r^{2}}\left(\frac{1}{r} \frac{\partial w}{\partial r}+\frac{1}{r^{2}} \frac{\partial^{2} w}{\partial \theta^{2}}\right) \\
& -2 \frac{\partial}{\partial r}\left(\frac{1}{r} \frac{\partial \chi}{\partial \theta}\right) \frac{\partial}{\partial r}\left(\frac{1}{r} \frac{\partial w}{\partial \theta}\right)
\end{aligned}
$$

The bilaplacian is such that $\nabla^{4} w=\nabla^{2} \nabla^{2} w$, and finally the Laplacian in polar coordinate is $\nabla^{2} w=\frac{1}{r} \frac{\partial}{\partial r}\left(r \frac{\partial w}{\partial r}\right)+$ $\frac{1}{r^{2}} \frac{\partial^{2} w}{\partial \theta^{2}}$. Notice that in the stress equation Eq.2, the operator $L(w, w)$ is often assumed to be equal to the Gaussian curvature of the surface characterized by $w(r, \theta)$, which is a true statement only in the limit where $\left|\frac{d w}{d r}\right|$ is small [5]. Once the Airy stress function is found as a solution of FvK equations, the planar stress components are computed in polar coordinate as

$$
\begin{aligned}
\sigma_{r r} & =\frac{1}{r} \frac{\partial \chi}{\partial r}+\frac{1}{r^{2}} \frac{\partial^{2} \chi}{\partial \theta^{2}} \\
\sigma_{\theta \theta} & =\frac{\partial^{2} \chi}{\partial r^{2}} \\
\sigma_{r \theta} & =-\frac{\partial}{\partial r}\left(\frac{1}{r} \frac{\partial \chi}{\partial \theta}\right)
\end{aligned}
$$

Finally, the planar strain components are also computed inverting Hooke's law as

$$
\begin{aligned}
& u_{r r}=\frac{1}{Y}\left[\sigma_{r r}-\nu \sigma_{\theta \theta}\right] \\
& u_{\theta \theta}=\frac{1}{Y}\left[\sigma_{\theta \theta}-\nu \sigma_{r r}\right]
\end{aligned}
$$

with $\nu$ the Poisson ratio.

These equations have been widely used in the litterature in order to address various problems of thin plates elasticity. However, since these equations are based on the mechanics of continuous media, the configuration of building blocks making up the shell is not taken into account. This lack has been cured by Seung and Nelson in a seminal work, where they show that the presence of topological defects is included as additional source terms in the compatibility equation [5]. In this case, the equation Eq.2 has to be modified

$$
\frac{\nabla^{4} \chi}{Y}=s(r, \theta)-\frac{1}{2} L(w, w)
$$

where $s(r, \theta)$ is the defect density. Within the present work, we will mainly consider two types of defects: disclinations (positive and negative) and dislocations. In triangular lattices, such as those formed by many selfassembling proteins like viral capsid proteins, positive 

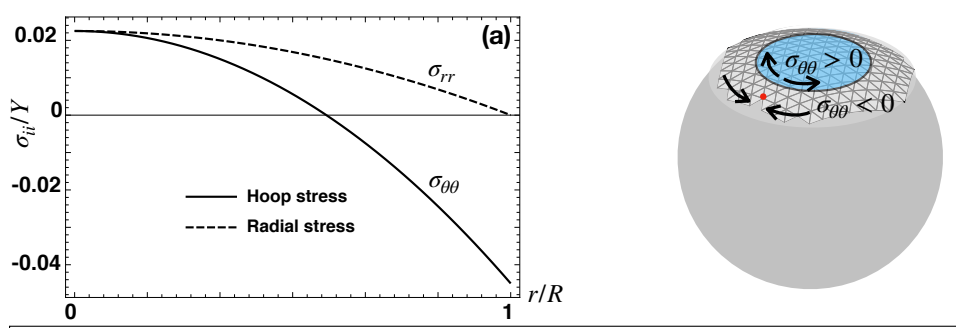

(b)

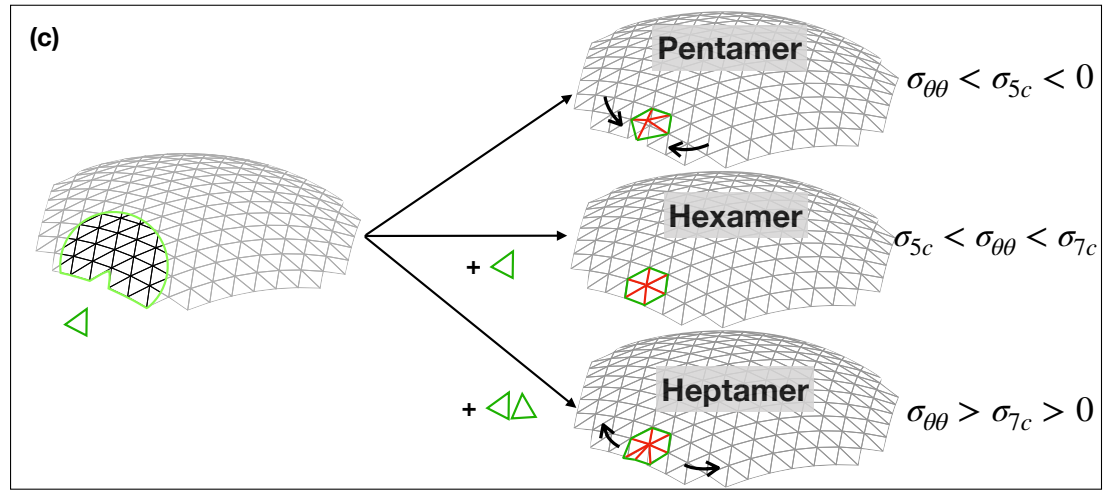

FIG. 1. Stresses during the self-assembly. The arrows indicate the direction of in-plane forces associated to the stress. (a) Variation of the hoop and radial stress for a self-assembling surface without defect. (b) Illustration of hoop stress variation across the hemisphere. (c) Proposed mechanism for defect nucleation illustrated on triangulated surfaces, based on the value of hoop stress. Threshold values for the hoop stress are labeled as $\sigma_{5 c}$ and $\sigma_{7 c}$. Parameters: $R / R_{0}=0.6$

disclinations are associated with proteins having five nearest neighbours, while negative disclinations are associated with seven neighbours. Within the context of continuous elasticity, the respective topological charges of these defects are $+\pi / 3$ and $-\pi / 3$. On the other hand, a single disclocation is associated to a bound pair of positive and negative disclinations, forming a structure similar to an electric dipole [24], and it is mainly characterized by its Burgers vector $\mathbf{b}$, whose amplitude is proportional to the triangular lattice step and its orientation is orthogonal to the line joining the two opposite disclinations. Following Seung et al., the defect density is written as

$$
s(\mathbf{r})=\sum_{\alpha} q_{\alpha} \frac{\pi}{3} \delta^{(2)}\left(\mathbf{r}-\mathbf{r}_{\alpha}\right)+\sum_{\beta} \epsilon_{i j} b_{j}^{\beta} \partial_{j} \delta^{(2)}\left(\mathbf{r}-\mathbf{r}_{\beta}\right)
$$

where $q_{\alpha}= \pm 1$ are the topological charges of the set of disclinations considered, and $\mathbf{b}^{\beta}$ are the Burgers vectors associated to the dislocation set.

In order to describe the growth of self-assembling proteins for example on a spherical surface, we will use the FvK equilibrium equations, together with the constraint that the growth occurs on the surface of a sphere. This imposes a deformation $w(r, \theta)$ such that $L(w, w) / 2=$ $K=1 / R_{0}^{2}$ is constant, corresponding to a constant Gaussian curvature. This condition is easily integrated to obtain $\frac{d w}{d r}=\sqrt{\alpha+K r^{2}}$. One further simplification of this profile is obtained by letting $\alpha=0$, which imposes a vanishing slope for the profile at the origin $\frac{d w}{d r}=r / R_{0}$. With this imposed deformation, it is possible to solve the stress equation Eq.11, with free boundary conditions $\sigma_{r r}(r=R, \theta)=0=\sigma_{r \theta}(r=R, \theta)$. Due to the linearity of the equation, the solution is found by considering the two source terms separately. Considering first the case of a spherical surface without defects, the constant positive Gaussian curvature leads to the following classical solutions for the Airy stress function, radial and azimuthal (or hoop) stress:

$$
\begin{aligned}
\chi_{0}(r) & =\frac{Y R^{4}}{64 R_{0}^{2}}\left(2\left(\frac{r}{R}\right)^{2}-\left(\frac{r}{R}\right)^{4}\right) \\
\sigma_{r r, 0} & =\frac{Y R^{2}}{16 R_{0}^{2}}\left(1-\left(\frac{r}{R}\right)^{2}\right) \\
\sigma_{\theta \theta, 0} & =\frac{Y R^{2}}{16 R_{0}^{2}}\left(1-3\left(\frac{r}{R}\right)^{2}\right)
\end{aligned}
$$

It was observed by many authors [19-21] that, while the radial stress is always positive, the hoop stress is positive close the center of the hemisphere, and negative as $r / R>1 / \sqrt{3}$. A positive stress is associated to a tensile region of the surface, while a negative one is associated to a compressed region. In the latter region, the molecules composing the surface are therefore subjected to azimuthal in-plane compression. This state of stress, which is also associated to a negative planar hoop strain, is prone to an out-of plane buckling instability [21], and it is at the origin of the wrinkling observed when a flat disk is forced to adhere on a sphere. The wrinkling instability is possible when the adhesion to the sphere is not strong enough, permitting therefore an out-of-plane deformation. Wrinkling has also been shown to relax the hoop stress both at a local and global scale. The nega- 
tive hoop stress region has been used also to discuss defect distributions in compression-free models, assuming further axisymmetry for the surface [19-21].

In the context of self-assembly, we anticipate that the negative hoop stress has very important consequences on the building up of local contacts between molecules at the rim line of the assembly (see figure 1). Indeed, considering a local arrangement of five subunits, awaiting for another subunit to eventually close into an hexagon, the negative hoop strain reduces strongly the space available for subunit addition, and closing the structure into a pentagon might become more likely, therefore leading to the nucleation of a fivefold defect of positive topological charge. Notice that the same argument could be reversed and applied to the nucleation of a sevenfold defect of negative charge: if the hoop stress is positive and it reaches a critical threshold, the large positive tension and strain on a local arrangement of five subunits might be enough to incorporate up to two subunits, therebye creating a sevenfold defect.

In order to estimate more quantitatively the mechanical influence of defect inclusion as the surface grows, we need to solve the Airy stress equation in the presence of defects for a positive constant Gaussian curvature. The derivation of the solution to this equation has been recently proposed by Grason [25, 26] for an arbitrary defect distribution. In this case, the Airy stress function associated to each defect has two contributions: a direct contribution which would exist for an infinite system, and an indirect contribution associated to the presence of a finite-size boundary. The latter contribution is equivalent to the inclusion of an image charge outside of the boundary. Following this derivation, we obtained lengthy expressions for the exact Airy stress function, hoop stress and radial stress, that are reported in Appendix A. With these expressions, we can observe quantitatively the influence of fivefold defect inclusion on stress relaxation, as it is shown in figure 2. Focusing on hoop stress evaluated at the rim $r=R$, we obtain a simpler expression for a single disclination of charge $s$ located at $\left(\rho_{1}, \theta_{1}\right)$ :

$$
\sigma_{\theta \theta, 1}(R, \theta)=\frac{Y s}{8 \pi} \frac{2\left(R^{2}-\rho_{1}^{2}\right)^{2}}{R^{2}\left(R^{2}+\rho_{1}^{2}-2 R \rho_{1} \cos \left(\theta-\theta_{1}\right)\right)}
$$

Assuming the existence of a negative threshold value $\sigma_{5 c}$ for the hoop stress represented as a red line in the figure, the inclusion of a single defect relieves the hoop stress at the rim in an anisotropic fashion: the effect is larger close the defect, where the stress becomes positive and therefore tensile, and it reduces for diametrically opposed points, where the initial negative stress is still present. In fact, defect inclusion changes the stress not only at the rim, but throughout the whole surface because of the long range nature of elastic potentials (see inset of figure 1a). However, as far as self-assembly is concerned, the modulation of hoop stress is mostly relevant at the rim of the growing surface, as it is the only location having degrees of freedom in order to relax the stress in an irreversible assembly. Increasing the size of the surface, it is observed that placing defects where the hoop stress is the most negative and beyond threshold $\sigma_{5 c}$ relaxes significantly the compressive hoop stress. In figure 2c for example, three defects relieve the stress efficiently for three quarters of the rim, but significant compressive hoop stress is still present in the last quarter, suggesting the optimal location of the next defect, in agreement with Li's analytical and numerical results [18]. Iterating this type of minimal hoop stress analysis, it is easy to realize that the azimutal location of defects is compatible with icosahedral symmetry. The elastic model is therefore able to predict the optimal directions for defect inclusion. Note that the radial position of the defect might however depend on further details of the model, like the contact energy between subunits.

Inspecting further the variation of hoop stress across the growing surface in the presence of fivefold defects, one realizes that other types of defect might also be nucleated in order to relax the mechanical stress. Indeed, as it is clearly observed in the inset of figure 1a, the presence of a single fivefold defect close to the rim induces a large positive hoop stress between the defect and the rim, along a radius of the disc. As a consequence, this burst of tensile (positive) hoop stress is prone to nucleate a sevenfold defect very close to the fivefold defect. In the continuous limit, this pair of defects is equivalent to a dislocation. Based on this observation, one expects that one or several dislocations might also be efficient at relieving the stress. Combining linearly the solution for the stresses associated to a positive and a negative disclination in the limit of vanishing separation, one obtains analytically the stresses for a single dislocation. The result is shown in Appendix A. Considering a single dislocation located at $\left(\rho_{1}, \theta_{1}\right)$, with an azimutal Burgers vector $\mathbf{b}=b \mathbf{e}_{\theta_{1}}$, the hoop stress evaluated at the rim is:

$$
\begin{aligned}
& \sigma_{\theta \theta, 1 d}(R, \theta)= \\
& \frac{Y b}{24} \frac{4\left(R^{2}-\rho_{1}^{2}\right)\left[\rho_{1}\left(3 R^{2}+\rho_{1}^{2}\right)-R\left(R^{2}+3 \rho_{1}^{2}\right) \cos \left(\theta-\theta_{1}\right)\right]}{R^{2}\left(R^{2}+\rho_{1}^{2}-2 R \rho_{1} \cos \left(\theta-\theta_{1}\right)\right)^{2}}
\end{aligned}
$$

Within such a dislocation, the equivalent fivefold and sevenfold disclination are located along a radius for the disc, the fivefold defect being closer to the center of the disc. In figure $3 \mathrm{a}$, several defects were compared, with respect to their ability to relax hoop stress along the rim of the assembly: a single disclination, a single dislocation, a dislocation plus a disclination, a small grain boundary (three dislocations in a row) and a small grain boundary terminated by a disclination. For a given size of the disc, it is observed that all the configurations produce similar pattern of hoop stress relaxation at the rim of the surface: close to the defect, hoop stress is relieved and becomes positive, while this stress relief decreases while reaching positions diametrically opposed to the $\operatorname{defect}(\mathrm{s})$. On the other hand, keeping the same defect configurations, and increasing the size of the disc as in figure $3 \mathrm{~b}$, we observe that the range of stress relaxation presents strong 

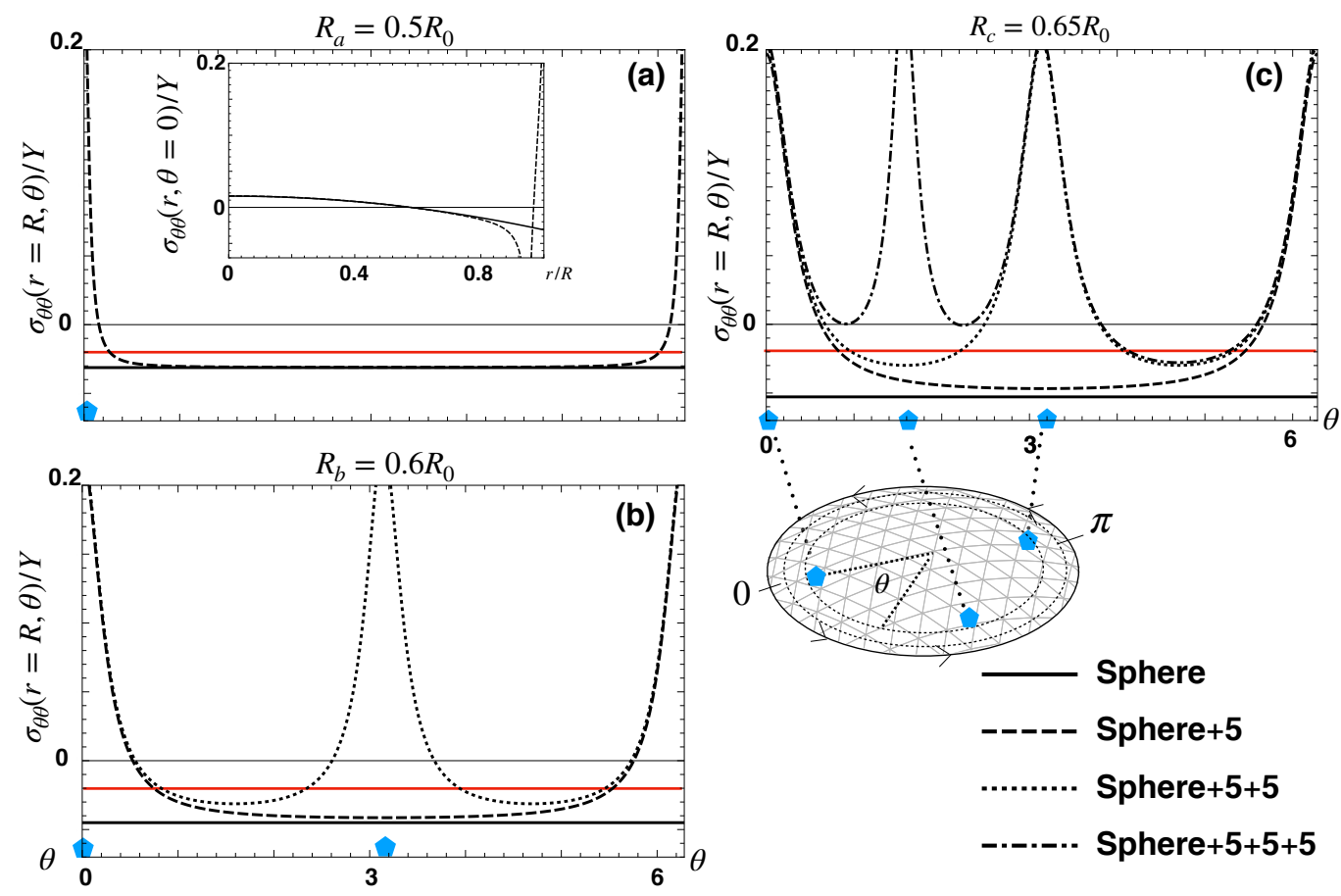

FIG. 2. Relaxation of hoop stress by defect nucleation. The hoop stress at the rim of the assembly is computed with (various dashed lines, see legend on figure) and without defects (line) as the surface grows ( $a$ to $c$ ). The size of the surface are shown on top of each graph. (a) Comparison of hoop stress without and with a single disclination (dashed line, $\rho_{1}=0.95 R_{a}$ ) as function of azimuthal angle. Inset: Hoop stress as function of radius. (b) A second disclination is added at the azimuthal location of smallest hoop stress, the radial position is $\rho_{2}=0.8 R_{b}$ (c) A third disclination is added at the azimuthal location of smallest hoop stress, the radial position is $\rho_{3}=0.85 R_{c}$. The defect radial locations are chosen in order to highlight the generic nature of anisotropic stress relaxation, but their precise location does alter the conclusion from this figure. The red line indicates a possible hoop stress threshold $\sigma_{5 c}$ for defect nucleation.

variation among defects configuration. For example, the combination of a grain boundary and a disclination provides a more efficient long range stress relief at the rim than a single disclination. This observation is compatible with the presence of grain boundaries in the ground state energy of closed shell for large radii previously mentioned in several works [6]. Our work is therefore able to provide an assembly pathway leading to the observed ground state for a closed shell.

It is possible to test the predictions of the elastic analytical model by using a standard numerical triangulated surface [5]. In this numerical computation, the structure is constructed using a triangular network reproducing the topological properties of the protein surface: most vertices are shared among six triangles, and at some locations they are shared among five or seven triangles, reproducing respectively positive and negative disclinations. A single dislocation is obtained by having two vertices of coordination five and seven nearest neighbours. The elastic energy is composed of two terms: an in-plane energy, associated with compression or stretching of triangles within their own plane, and out-of-plane bending energy. The total elastic energy is therefore written as

$$
E_{t o t}=\frac{k_{e}}{2} \sum_{\alpha, \beta}\left(d_{\alpha, \beta}-d_{0}\right)^{2}+k_{b} \sum_{i, j}\left(1-\cos \left(\theta_{i, j}-\theta_{0}\right)\right)
$$

where $d_{\alpha, \beta}$ is the length of the edge relating vertices $\alpha$ and $\beta$, and $\theta_{i, j}$ is the angle between the normal vectors to triangles $i$ and $j$. The remaining set of parameters $\left\{k_{e}, k_{b}, d_{0}, \theta_{0}\right\}$ defines the intrinsic elastic properties of the building blocks. In particular, the spontaneous angle $\theta_{0}$ is associated with a radius of spontaneous curvature of order $R_{0} \sim d_{0} / \theta_{0}$. The continuous limit of this discrete model corresponds to the analytical model discussed so far provided that the elastic constants $k_{e}, k_{b}$ are related to Young modulus and flexural rigidity with the following relations $Y=2 k_{e} / \sqrt{3}$ and $D=\sqrt{3} k_{b} / 2$ [5]. The triangular lattice with appropriate defect locations is constructed by putting each vertex on the surface of a sphere of radius $R_{0}$, and the final structure is relaxed by minimizing the energy using a conjugate gradient method. Notice that we added a radial Morse potential between the vertices and the surface of the sphere, in order to force the triangular structure to adhere on the sphere during 


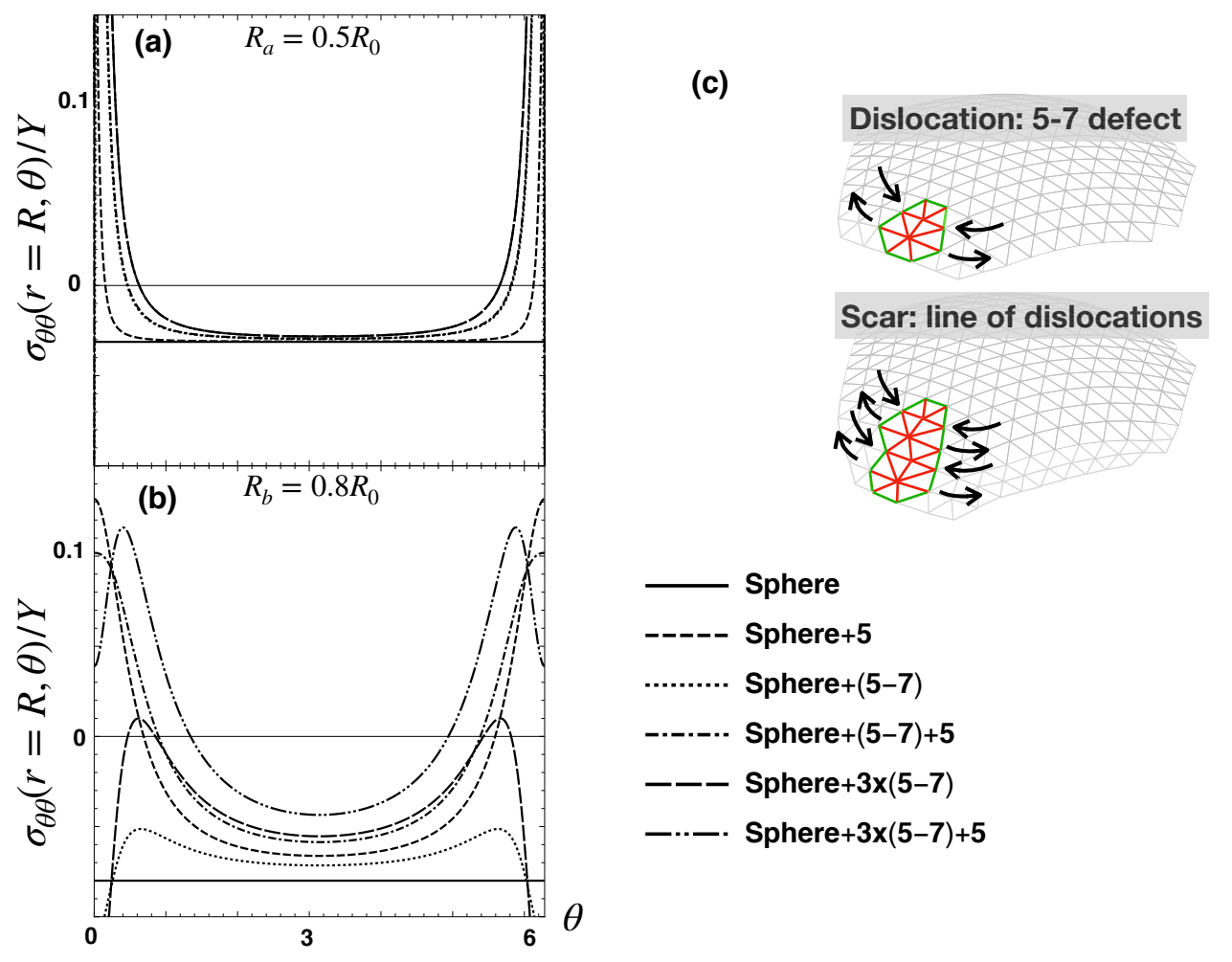

FIG. 3. Short and long range relaxation of hoop stress using different defect configurations. These configurations are used to relax partially mechanical stress at a given size $R_{a}$ (a), and their effect is shown if growth persists until $R_{b}$ without further inclusion of defects (b). The size of the surface are shown on top of each graph. All defects are arranged along a single radius of the disc. Their radial positions are as follows: isolated defects $\rho_{5}=\rho_{57}=0.95 R_{a}$. Combined dislocation (5-7) and disclination $(+5)$ are arranged as follows $\rho_{57}=0.95 R_{a}$ and $\rho_{5}=0.97 R_{a}$. Combined dislocations are arranged as follows: $\rho_{57}^{(1)}=0.95 R_{a}, \rho_{57}^{(2)}=0.97 R_{a}, \rho_{57}^{(3)}=0.99 R_{a}$, and final disclination is at $\rho_{5}=0.996 R_{a}$. Although the defect configurations seem equivalent in order to relax mechanical stress for $R_{a}$, strong modulation of relaxing effect is observed at large scale (b). (c) Typical configurations at the rim of the triangulated surface: a single dislocation (5-7) and a single scar made of two dislocations. The red links are connected either to fivefold or sevenfold vertices.

the energy minimization. Provided that the adhesion is strong enough, we checked that the purely elastic energy of this discrete hemisphere do not depend on the parameters used for Morse potential.

We chose to compare the elastic energy density at the rim of the surface within analytical and numerical models. In both cases, we omit the bending energy since it is expected to be homogeneous. In the analytical case, it is known that the stretching energy of a surface element $d S$ is given by $d S\left(\sigma_{\theta \theta}+\sigma_{r r}\right)^{2} /(2 Y)=d S \sigma_{\theta \theta}^{2} /(2 Y)$, when evaluated at the rim of the surface. In the numerical case, the energy density $e_{l o c a l}$ is estimated by averaging, for each vertex of the rim, the stretching energy of edges connected to that vertex. The result of the quantitative comparison is shown in figure 4 . In the case of a single defect (disclination or dislocation), the analytical and numerical energy densities show a satisfactory quantitative agreement. Remarkably, no parameter adjustment has been used to obtain this agreement. When the comparison is performed on a multi-disclination configuration, the agreement is also remarkable. Overall, these exam- ples of comparison validate the analytical result obtained in this work.

\section{DISCUSSION}

Curved 2D crystals are known to exhibit geometric frustration, i.e. the long range configuration imposed by the curvature is not compatible with the short range arrangement of its building blocks. This frustration is usually accommodated by having topological defects embedded in their structure. Raising the question of how and where these defects will arise in self-assembled systems where each building blocks are identical, we proposed in this work an analytical elastic model in order to quantify the mechanical stress and the assembly pathway on a spherical geometry. Our analysis led us to identify the in-plane hoop stress evaluated at the rim of the hemispherical cap as the most relevant quantity in order to explain defect nucleation. Indeed, in the scenario considered, the rim of the assembly is the only degree of freedom being able to reduce the geometric frustration 

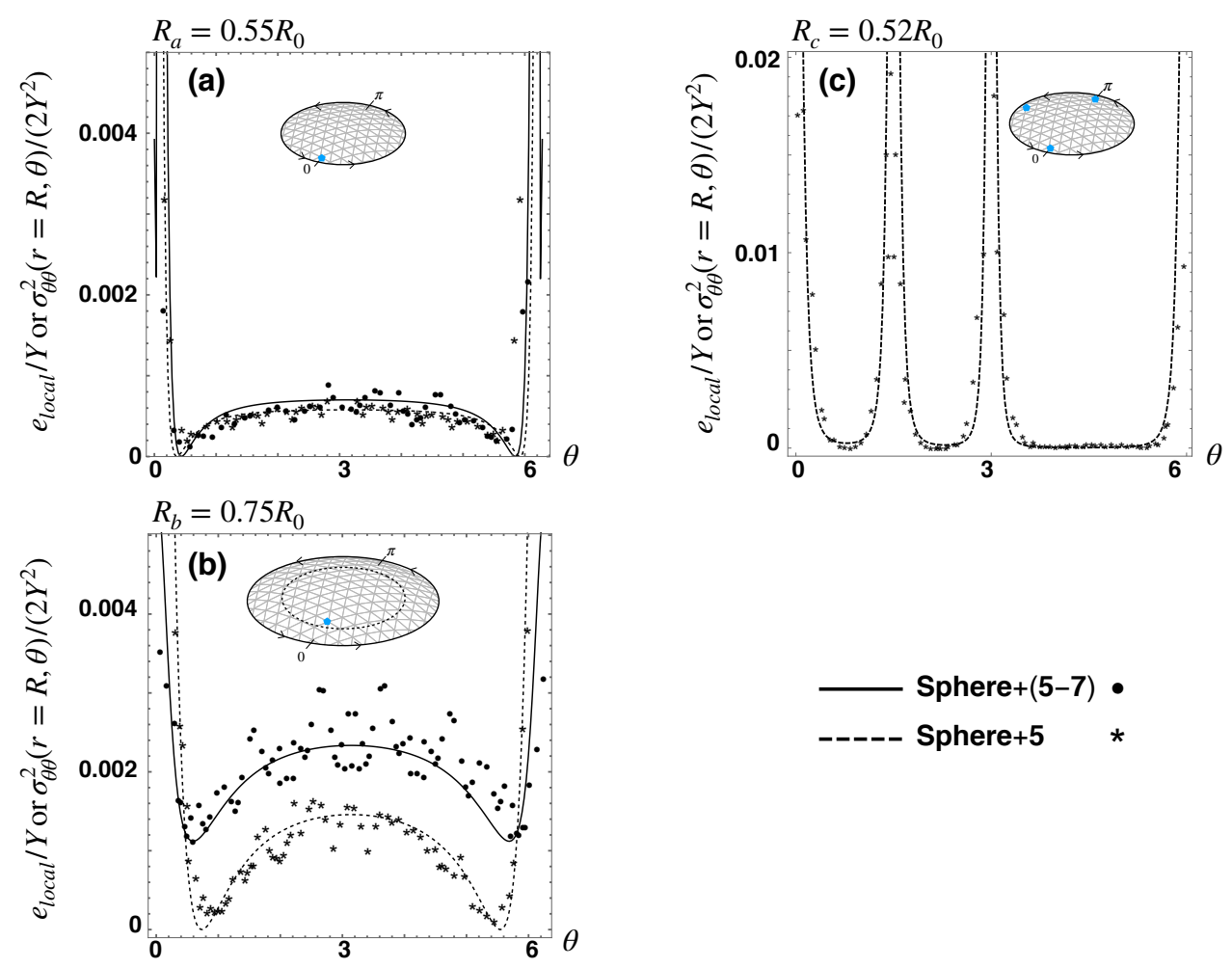

FIG. 4. Comparison of energy densities at the rim between analytical (lines) and numerical (symbols) calculations. The case of a single disclination (star and dashed line) or a dislocation (dot and thin line) is shown for two sizes: (a) $R_{a}=0.55 R_{0}$ and (b) $R_{a}=0.75 R_{0}$. (c) Three disclinations configuration with $90^{\circ}$ relative orientation. Other parameters: $R_{c}=0.52 R_{0}$, $\rho_{5}^{(a)}=\rho_{57}^{(a)}=0.88 R_{a}, \rho_{5}^{(1,2,3)}=0.88 R_{c}$.

of the curved crystal. This inherent frustration is associated to a transition of the hoop stress from a tensile to a compressive state, the latter being prone to buckling (out of plane) phenomena. Computing analytically this value of hoop stress for different disclination configurations, we showed that defect nucleation is able to relax locally the compressive stress at the rim, and therefore it might ease further assembly. We also showed that this stress relief is anisotropic, suggesting preferred directions for successive defect nucleations. These results are complementary to those of compression-free models [19-21], in which symmetric defect distributions are seeked in order to vanish the hoop stress in an entire region. Within our work, we focused on the self-assembly process and therefore we investigated the modulation of hoop stress at the rim. Non-axisymmetric hoop stress calculations allowed us to go beyond compression-free models at the rim in that case.

Moreover, we computed the stress relaxation pattern at the rim for more complex defect configurations. In particular, our results confirm that radial lines of dislocations, known as scars, are also efficient at relaxing mechanical stress. This quantitative observation suggests for the first time that the ground state of large closed spherical surfaces with crystalline order can be reached in self-assembling systems thanks to the proposed me- chanical stress relaxation mechanism. Indeed, it has been shown for these systems, both experimentally and theoretically, that the ground state is a superimposition of twelve fivefold disclinations decorated by multiple scars. This particular geometry can be therefore reached as the system is self-assembled, rather than by reorganizing locally the structure in a system with an initial idealized icosahedral symmetry, which might be a process that would require more energy.

All the defect configurations that we tested in this work have a common trait: defects are arranged along a single radial direction. This simplifying assumption is discussed and justified in the supplemental material file by comparing single to multiple directions for the defect distributions. Indeed, we observed that choosing multiple directions in order to relax the stress has an amplifying effect, but its does not change the picture drawn from single direction defect distribution. Moreover, we analyzed also in the supplemental material file the scar length dependence of stress relaxation. In this case, it is observed that best relaxation efficiency is obtained with increasing scar length. Combining the stress relaxation ability of scar length and scar numbers observed in figure 1 and 2 of supplemental material, we draw the following conclusion: different defect configurations are able to induce similar stress relaxation pattern, and in particular 
if a larger number of scars is used in order to relax the stress, their lengths have to be reduced in order to reach a similar level of stress relaxation. Within our model, which is based on the analysis of stress distribution, we did not find some objective way to determine the actual defect distribution. Rather, it is likely that the precise distribution is determined by computing the elastic energy for each configurations and optimizing it, like in the work of Li et al. [18]. Therefore analyzing the stress pattern allows mainly to determine the different mechanisms of defect nucleation.

The mechanism of defect nucleation through hoop stress relaxation at the rim proposed in this work introduces two hoop stress thresholds $\sigma_{5 c}$ and $\sigma_{7 c}$ for pentamer and heptamer nucleation respectively. Although their scaling with physical parameters of the elastic model is expected to be similar, the precise values of these threshold is expected to be model-dependent. We provided for example in an appendix a scaling estimate of $\sigma_{5 c}$, which mainly depends on the adhesion energy between assembling subunits, Young modulus and shell thickness. In order to test the influence of these threshold parameters on the nucleation of defects, we provided additional simulation results in the supplemental material file. Within these simulations, it is observed that decreasing the heptamer threshold $\sigma_{7 c}$ at fixed pentamer threshold $\sigma_{5 c}$ allows to modify the early steps of defect nucleation: for large $\sigma_{7 c}$, the first defect to be nucleated are pentamers. This result is not really new as it has been observed by many groups $[9,18]$. However, if $\sigma_{7 c}$ is reduced, the first defects to be nucleated are dislocations or a mix of disclination and dislocation. This is a new result, as previous simulations were mainly designed to monitor the appearance of pentamers. Reducing further the threshold shifts the defect nucleation to scar-dominated regime. Our model and algorithm is therefore able to take into account more complex defect distributions.

In order to highlight the role of mechanical stress in the nucleation of defects, we chose a spherical surface as an idealized geometry for the sake calculation tractability. However, the qualitative features of the results demonstrated analytically for this particular geometry are expected to be valid for various scaffolding surface. The main reason is that the Gaussian curvature of the growing surface is a source for in-plane stress. A given distribution of Gaussian curvature will therefore generate a particular stress pattern at the rim where the assembly is effectively proceeding. Nucleating a fivefold defect will reduce locally along the rim the mechanical stress. Depending on the particular Gaussian curvature profile, we expect to generate an appropriate distribution of defects, including fivefold and sevenfold defects and/or one or several dislocations. For example, for geometries with negative Gaussian curvature we anticipate that sevenfold defects are expected to be most relevant in relaxing mechanical stress, which should now be tensile in excess at the rim [17].

One important assumption in the present model is that the growth of the structure is irreversible, in the sense that the local structure of the surface is determined by the rim of the assembly. This neglects any further rearrangement away from the rim in the bulk of structure that has been assembled. These rearrangements represent another degree of freedom that could be used in order to relax the mechanical stress. However, their contribution might not be as important for two reasons. First, single disclination motion requires large scale rearrangement and are therefore unlikely [25]. On the other hand, dislocation motion are known to require mostly local structural changes, and this is the cost for these changes that will determine if the structure can relax using dislocation motion [27-29]. Roughly, if the interaction between subunits is strong enough, bulk relaxation becomes again unlikely because of the high cost for individual dislocation motion, leaving defect nucleation at the rim as the only effective way of reducing mechanical stress during the assembly. Notice that the particular geometrical configuration of the subunit at the rim of the assembly favors defect nucleation rather than bulk defect mobility, whatever the physical parameters. This qualitative analysis is consistent with the recent work by Panahandeh et al. who compared directly the phase diagram of irreversible and relaxed self-assembly [30]. They found that for most of the phase diagram, the structures are very similar. This confirms that the main structural features, including defect position is indeed determined at the rim of the assembly.

We note also that the azimutal position of defects as the surface grows predicted by searching the most negative hoop stress values at the rim is consistent with results obtained by minimizing the global energy of the surface as the surface is assembled [18], at least for the nucleation of the firsts defects. The two models might differ at larger scale though because Li's model is solved in true spherical coordinates, while ours is solved using polar coordinates for the sake of tractibility and it is therefore expected to only apply for structures with moderate deviations from planar configuration, but the physical ingredients are essentially the same.

We hope that the present work will motivate future investigations, extending the present model in order to describe defect nucleation until full completion of the spherical surface, or to describe the interplay between defect nucleation and rearrangement. Additional data are provided as supplemental material file.

Acknowledgments Stimulating discussions with R. Zandi, B. Dragnea, B. Davidovitch and M. Adda-Bedia are greatfully acknowledged at various steps of the work. This work was partly funded through the CNRS-PEPS program entitled "Modeling infectious processes". 


\section{APPENDIX: SOLUTION OF AIRY STRESS EQUATION}

The derivation of the solution to the Airy stress equation in the presence of a single defect of topological charge $s= \pm \pi / 3$ located at $\left(r_{d}=\rho, \theta_{d}=0\right)$ has been provided in Grason's work [25]. We present here only the result for the complete Airy stress function, hoop and radial stress, which have not been provided in that reference. The idea of the calculation is to decompose the stress into two parts: the first part is the solution of the equation without boundary, and the second part takes into account the stress induced by the boundary. It is formally equivalent to use an image charge outside the boundary in order to enforce the free boundary conditions. Defining the distance between the point where the stress function is evaluated $(r, \theta)$ and the defect location $(\rho, 0)$ as $\hat{r}^{2}=r^{2}+\rho^{2}-2 r \rho \cos \theta$, the direct and indirect contributions are written respectively as

$$
\begin{aligned}
\frac{\chi_{1 d}}{Y s /(8 \pi)} & =\hat{r}^{2}\left[\log \rho-\sum_{n=1}^{\infty} \frac{1}{n}\left(\frac{r}{\rho}\right)^{n} \cos (n \theta)\right] \text { if } r<\rho \\
& =\hat{r}^{2}\left[\log r-\sum_{n=1}^{\infty} \frac{1}{n}\left(\frac{\rho}{r}\right)^{n} \cos (n \theta)\right] \text { if } r>\rho \\
\frac{\chi_{1 i}}{Y s /(8 \pi)} & =\sum_{n=0}^{\infty}\left[C_{n} r^{n}+D_{n} r^{n+2}\right] \cos (n \theta)
\end{aligned}
$$

The coefficients $C_{n}$ and $D_{n}$ are chosen in order to satisfy the boundary conditions $\sigma_{r r}(r=R, \theta)=0=\sigma_{r \theta}(r=$ $R, \theta)$. Once these coefficients have been found, exact summations are used to obtain an exact expression of Airy stress function. The image charge is located outside the boundary at a radial distance $\hat{\rho}$ such that $\rho \hat{\rho}=R^{2}$. Introducing the distance between the point of observation and the image charge ${\hat{r_{i}}}^{2}=r^{2}+\hat{\rho}^{2}-2 r \hat{\rho} \cos \theta$, the result can be rewritten as:

$$
\begin{aligned}
\frac{\chi_{1}}{Y s /(8 \pi)}= & \frac{\hat{r}^{2}}{2} \log \left[\frac{\hat{r}^{2} R^{4}}{{\hat{r_{i}}}^{2} \rho^{2}}\right] \\
& -\frac{r\left(2 \rho^{3} \cos \theta+r\left(R^{2}-\rho^{2}+R^{2} \log \left[R^{2}\right]\right)\right)}{2 R^{2}}
\end{aligned}
$$

Using the definition of Airy stress function in polar coordinate, we obtain the hoop and radial stress as:

$$
\begin{aligned}
\frac{\sigma_{\theta \theta, 1}}{Y s /(8 \pi)}= & -1+\frac{\rho^{2}}{R^{2}}+\log \left[\frac{\hat{r}^{2} R^{2}}{\hat{r}_{i}^{2} \rho^{2}}\right] \\
& +\frac{3 r^{2}+\rho^{2}+2 \rho \cos \theta(\rho \cos \theta-3 r)}{\hat{r}^{2}}-\frac{B}{\hat{r}_{i}^{4} \rho^{4}} \\
B= & \left(\hat{r}_{i}^{2} \rho^{3}\right)\left[5 r^{2} \rho+\rho^{3}-2 r \cos \theta\left(2 R^{2}+3 \rho^{2}\right)\right. \\
& \left.+4 R^{2} \rho(\cos \theta)^{2}\right]+2 \rho^{2} \hat{r}^{2}\left(r \rho-R^{2} \cos \theta\right)^{2} \\
\frac{\sigma_{r r, 1}}{Y s /(8 \pi)}= & \frac{\rho^{2}}{R^{2}}+\log \left[\frac{\hat{r}^{2} R^{2}}{\hat{r}_{i}^{2} \rho^{2}}\right]+\frac{2 \rho^{2}(\sin \theta)^{2}}{\hat{r}^{2}}-\frac{A}{\hat{r}_{i}^{4} \rho^{2}}(22) \\
A= & 2 R^{6}+r^{2} \rho^{2}\left(r^{2}+4 R^{2}\right)+r^{2} \rho^{4} \\
& -r \rho \cos \theta\left[3 R^{4}+2 R^{2} \rho^{2}+2 r^{2}\left(R^{2}+\rho^{2}\right)\right] \\
& +R^{4}\left[\cos (2 \theta)\left(r^{2}-2 R^{2}+\rho^{2}\right)+r \rho \cos (3 \theta)\right]
\end{aligned}
$$

In particular, for increasing subunit-subunit adhesion energy, the critical hoop stress is more negative, reflecting larger resistance to in-plane compression.

[1] Mateu MG. Assembly, stability and dynamics of virus capsids. Arch Biochem Biophys. 2013;531:65.

[2] Hinzpeter F, Gerland U, Tostevin F. Optimal compartmentalization strategies for metabolic microcompartments. Biophys J. 2017;112:767.

[3] Caspar DL, Klug A. Physical principles in the construction of regular viruses. Cold Spring Harbor Symp Quant Biol. 1962;27:1. 
[4] Zandi R, Reguera D, Bruinsma RF, Gelbart WM, Rudnick J. Origin of icosahedral symmetry in viruses. Proc Nat Acad Sci. 2004;101:15556.

[5] Seung HS, Nelson DR. Defects in flexible membranes with crystalline order. Phys Rev A. 1988;38:1005.

[6] Bowick M, Nelson DR, Travesset A. Interacting topological defects on frozen topographies. Phys Rev B. 2000;62:8738

[7] Vitelli V, Lucks JB, Nelson DR. Crystallography on curved surfaces. Proc Nat Acad sci. 2006;103:12323.

[8] Giomi L, Bowick M. Crystalline order on Riemannian manifolds with variable Gaussian curvature and boundary. Phys Rev B. 2007;76:054106.

[9] Hicks SD, Henley CL. Irreversible growth model for virus capsid assembly. Phys Rev E. 2006;74:031912.

[10] Olson A, Hu YHE, Keinan E. Chemical mimicry of viral capsid self-assembly. Proc Nat Acad Sci. 2007;104:20731.

[11] Levandovsky A, Zandi R. Non-equilibrium assembly, retroviruses and conical shape. Phys Rev Lett. 2009;102:198102.

[12] Wagner J, Zandi R. The robust assembly of small symmetric nanoshells. Biophys J. 2015;109:956.

[13] Grime JMA, Dama JF, Ganser-Pornillos BK, Woodward CL, Yeager GJJM, Voth GA. Coarse-grained simulation reveals key features of HIV-1 capsid self-assembly. Nat Comm. 2015;7:11568.

[14] Castelnovo M. Viral self-assembly pathway and mechanical stress relaxation. Phys Rev E. 2017;95:052405.

[15] Dinsmore AD, Hsu MF, Nikolaides MG, Marquez M, Bausch AR, Weitz DA. Colloidosomes: selectively permeable capsules composed of colloidal particles. Science. 2002;298:1006.

[16] Bausch AR, Bowick MJ, Cacciuto A, Dinsmore AD, Hsu MF, Nelson DR, et al. Grain boundary scars and spherical crystallography. Science. 2003;299:1716.

[17] Irvine WTM, Vitelli V, Chaikin PM. Pleats in crystals on curved surfaces. Nature. 2010;468:947.
[18] Li S, Roy P, Travesset A, Zandi R. Why large icoshedral viruses need scaffolding proteins. Proc Nat Acad Sci. 2018;115:10971.

[19] Azadi A, Grason GM. Emergent structure of multidislocation ground states in curved crystals. Phys Rev Lett. 2014;112:225502.

[20] Azadi A, Grason GM. Neutral versus charged defect patterns in curved crystals. Phys Rev E. 2016;94:013003.

[21] Grason GM, Davidovitch B. Universal collapse of stress and wrinkle-to-scar transition in spherically confined crystalline sheets. Proc Nat Acad Sci. 2013;110:12893.

[22] Landau LD, Lifshitz EM. Theory of elasticity. Pergamon press; 1970.

[23] Ventsel E, Krauthammer T. Thin plates and shells: theory, analysis and applications. CRC press; 2001.

[24] Vitelli V, Nelson DR. Defect generation and deconfinement on corrugated topographies. Phys Rev E. 2004;70:051105.

[25] Grason GM. Defects in crystalline packings of twisted filament bundles. I. Continuum theory of disclinations. Phys Rev E. 2012;85:031603.

[26] Azadi A, Grason GM. Defects in crystalline packings of twisted filament bundles. II. Dislocations and grain boundaries. Phys Rev E. 2012;85:031604.

[27] Falk ML. Molecular dynamics study of ductile and brittle fracture in model non crystalline solids. Phys Rev B. 1999;60:7062.

[28] Nabarro FRN. Theory of crystal dislocations. Oxford University Press; 1967.

[29] Hirth JP, Lothe J. Theory of dislocations. McGraw-Hill, New York; 1972.

[30] Panahandeh S, Li S, Zandi R. The equilibrium structure of self-assembled protein nano-cages. Nanoscale. 2018;10:22802. 